\title{
Reverse glycerol catabolism pathway for dihydroxyacetone and glycerol production by Klebsiella pneumoniae
}

\section{Shaoqi Sun}

Shanghai Advanced Research Institute

\section{Yike Wang}

Shanghai Advanced Research Institute

\section{Lin Shu}

Shanghai Advanced Research Institute

\section{Xiyang Lu}

Shanghai Advanced Research Institute

\section{Qinghui Wang}

Shanghai Advanced Research Institute

Chengang Zhu

Shanghai University

\section{Jiping Shi}

Shanghai Advanced Research Institute

\section{Gary J. Lye}

University College London

\section{Frank Baganz}

University College London

Jian Hao ( $\sim$ haoj@sari.ac.cn )

Shanghai Advanced Research Institute, Chinese Academy of Sciences https://orcid.org/0000-00024403-8188

\section{Research}

Keywords: Dihydroxyacetone, glycerol, tpiA, hdpA, Klebsiella pneumoniae

Posted Date: January 25th, 2021

DOI: https://doi.org/10.21203/rs.3.rs-152355/v1

License: (c) (1) This work is licensed under a Creative Commons Attribution 4.0 International License. Read Full License 


\section{Abstract}

Background: Klebsiella pneumoniae is a bacterium that can be used as producer for numerous chemicals. Glycerol can be catabolised by K. pneumoniae and dihydroxyacetone is an intermediate of this catabolism pathway. Here dihydroxyacetone and glycerol were produced from glucose by this bacterium based on a reverse glycerol catabolism pathway.

Results: tpiA, encoding triosephosphate isomerase, was knocked out to block the further catabolism of dihydroxyacetone phosphate in the glycolysis. After overexpression of a Corynebacterium glutamicum dihydroxyacetone phosphate dephosphorylase $(h d p A)$, the engineering strain produced remarkable levels of dihydroxyacetone and glycerol from glucose. Further increase in product formation were obtained by knocking out gapA encoding an iosenzyme of glyceraldehyde 3-phosphate dehydrogenase. There are two dihydroxyacetone kinases in K. pneumoniae. They were both disrupted to prevent an inefficient reaction cycle between dihydroxyacetone phosphate and dihydroxyacetone, and the resulting strains had a distinct improvement in dihydroxyacetone and glycerol production. pH 6.0 and low air supplement were identified as the optimal conditions for dihydroxyacetone and glycerol production by $K$, pneumoniae $\triangle$ tpiA- $\triangle D H A K-h d p A$. In fed batch fermentation $23.9 \mathrm{~g} / \mathrm{L}$ of dihydroxyacetone and $10.8 \mathrm{~g} / \mathrm{L}$ of glycerol were produced after 91 hours of cultivation, with the total conversion ratio of $0.97 \mathrm{~mol} / \mathrm{mol}$ glucose.

Conclusions: This study provides a novel and highly efficient way of dihydroxyacetone and glycerol production from glucose.

\section{Background}

Klebsiella pneumoniae is a facultative anaerobic gram-negative rod-shaped bacterium and belongs to the family of Enterobacteriaceae. It is ubiquitous distributed in natural environments and can catabolise many kinds of carbon sources. K. pneumoniae is also an important industrial microorganism. Wild type strains have been used as producers for 1,3-propanediol and 2,3-butanediol production. After metabolic engineering modification, K. pneumoniae strains have been used as workhorses for 2-ketogluconic acid [1], gluconic acid [2], xylonic acid [3], acetoin [4], 2,3-dihydroxyisovalerate [5], 2-ketoisovalerate and isobutanol production [6].

Glycerol is a suitable carbon source for $K$. pneumoniae growth, and it was used for 1,3-propanediol production. The glycerol catabolism pathway is a dismutation process. In the oxidative branch, glycerol is oxidized to dihydroxyacetone (DHA) under the catalysis of glycerol dehydrogenase, and the latter is then phosphorylated to DHA phosphate and channeled into glycolysis. In a coupled reductive pathway, glycerol is converted to 3-hydroxypropionaldehyde and further converted to 1,3-propanediol [7]. Glycerol dehydrogenase, glycerol dehydrase and 1,3-propanediol dehydrogenase are encoded by dhaD, dhaB and $d h a T$, respectively. There are two DHA kinases in K. pneumoniae. DHA kinase I use ATP as the cofactor and DHA kinase II use phosphorenol pyruvate (PEP) as the cofactor [8]. DHA kinase I is encoded by dhaK. DHA kinase II consists of three subunits, and they are encoded by dhaK1, dhaK2 and dhaK3, respectively. 
Genes of those enzymes are in an operon named dha operon [9]. When glycerol is used as the carbon source, the main metabolites of $K$. pneumoniae are 1,3-propanediol, 2,3-butanediol and some organic acids [7].

Dihydroxyacetone (DHA) is a simple, achiral, and non-toxic sugar. DHA is a very important chemical product. It is used extensively in the cosmetic industry for making artificial suntans. DHA is used as a building block for the synthesis of lactic acid and 1,2-propanediol. DHA is also extensively used in the medical industry [10]. DHA can be synthesized through a chemical route with formaldehyde as the substrate, but the product is a mixture of many unbranched aldoses and ketoses [11]. DHA was efficienctly produced from glycerol through a biotransformation process by Gluconobacter oxydans. In $G$. oxydans, the enzyme responsible for the oxidation of glycerol to DHA is a membrane-bound glycerol dehydrogenase, which use pyrrolequinone as the cofactor [12]. A Pichia membranifaciens strain was reported for the production of DHA from glycerol, but the responsible enzymes were unidentified [13]. A mutant of Hansenula polymorpha was reported to produce dihydroxyacetone from methanol in a restingcells reaction. Methanol was catabolised through a xylulose monophosphate cycle pathway in this yeast [14].

Corynebacterium glutamicum has a metabolic pathway that convert sugars to glycerol via DHA as an intermediate, and this pathway has been used for 1,2-propanediol and 1-propanol producing strains construction [15]. DHA phosphate is an intermediate in the glycolysis and was converted to DHA by a $h d p A$ encoded DHA phosphate dephosphorylase. DHA was further converted to glycerol or converted to methylglyoxal for 1,2-propanediol production. A DHA producing $E$. coli strain was constructed through expression of the G. glutamicum hdpA gene [16].

DHA is an intermediate of the glycerol catabolism pathway in K. pneumoniae. But it cannot accumulate in the process. Cultivation of $K$. pneumoniae that use glucose or other sugars as the carbon source, produce as main metabolites 2,3-butanediol and some organic acids [17], glycerol or DHA were not metabolic products of this process. In this work, a reverse glycerol catabolic pathway was constructed in K. pneumoniae for DHA and glycerol production from glucose (Fig. 1).

\section{Results}

\section{Thermodynamic analysis of glycerol catabolic pathway}

Gibbs free energy $(\Delta \mathrm{rG})$ and equilibrium constant $\left(\mathrm{K}_{\text {eq }}\right)$ of reactions and pathways related to glycerol catabolism were estimated with the help of eQuilibrator, and results are given in Table 1.

DhaD catalyses the conversion of glycerol to DHA, and NAD is used as the cofactor (No. 1 in Table 1). The estimated $\Delta \mathrm{rG}$ of this reaction was positive, and $\mathrm{K}_{\text {eq }}$ was lower than 1 . It indicated that this reaction prefers glycerol formation rather than DHA formation. At reaction equilibrium condition, the level of DHA would be much lower than that of glycerol. 
DHA kinase I that is encoded by dhaK catalyses DHA phosphate (DHA-P) formation and this reaction use ATP as the phosphate donor (No. 2 in Table 1). The estimated $\Delta r G$ of this reaction was $-13.7 \pm 4.3$ $\mathrm{kJ} / \mathrm{mol}$. Combining this reaction with the reaction of DHA formation from glycerol that is catalysed by DhaD, the $\triangle \mathrm{rG}$ of this pathway (No. $1+2$ in Table 1) was $10.9 \mathrm{~kJ} / \mathrm{mol}$. Consider the levels of ATP and ADP in K. pneumoniae were nearly equal [18]. Thus, the pathway of glycerol catabolism that is catalysed by the corresponding two enzymes was not preferred from the thermodynamic aspect. In other words, the reverse pathway of glycerol formation from DHA phosphate was possible.

DHA kinase Il catalyses DHA phosphate formation with phosphoenolpyruvic acid (PEP) as the phosphate donor (No. 3 in Table 1). The estimated $\Delta r G$ of this reaction was $-41.4 \pm 4.4 \mathrm{~kJ} / \mathrm{mol}$, which was more negative than the reaction that is catalysed by DHA kinase I. Accordingly, the $\triangle \mathrm{rG}$ of the pathway that catalysed by DHA kinase II and DhaD (No. 1+3 in Table 1) was $-16.8 \mathrm{~kJ} / \mathrm{mol}$. This glycerol catabolism pathway was preferred from a thermodynamic aspect.

DHA phosphate dephosphorylase ( $h d p A)$ of $C$. glutamicum catalyses the reaction of DHA formation from DHA phosphate (No. 4 in Table 1). DHA phosphate was hydrolyzed to release free phosphate and DHA. This reaction was not linked with ATP or PEP formation. The $\Delta \mathrm{rG}$ of this reaction was $-12.7 \pm 4.3 \mathrm{~kJ} / \mathrm{mol}$. Combining this reaction with the $\mathrm{DhaD}$ catalysed reaction to set up a reverse glycerol catabolism pathway (No. 4-1 in Table 1), and the estimated $\Delta \mathrm{rG}$ of this pathway was $-37.3 \mathrm{~kJ} / \mathrm{mol}$. Then, DHA and glycerol formation from DHA phosphate through this pathway was feasible from a thermodynamic aspect.

\section{DHA production from DHA phosphate}

The glycerol catabolism pathway that formed by DhaD and Dhak (No. $1+2$ in Table 1 ) has a positive $\Delta \mathrm{rG}$. It indicated glycerol might be formed from DHA phosphate, which is an intermediate of the glycolysis. However, there are no reports of glycerol formation from glucose or other sugars by wild type $K$. pneumoniae.Kp $\triangle t$ tpiA was constructed to block the conversion of DHA phosphate to glyceraldehyde 3phosphate. This strain and the wild type of $K$. pneumoniae were cultured in flasks with glucose as the carbon source and results are shown in Fig. 2.

$23 \mathrm{~g} / \mathrm{L}$ glucose was consumed by the wild-type $K$. pneumoniae after 9 hours of cultivation. Cell growth was coincided with glucose consumption and $3.2 \pm 0.2$ OD units of cell density was achieved after glucose exhausted. The main metabolites of the process were 2,3-butanediol and acetoin, and their final titers were $3.6 \pm 0.5$ and $2.6 \pm 0.2 \mathrm{~g} / \mathrm{L}$, respectively. Low level of acetic acid and lactic acid were produced in the process (data not shown). Cell growth of $K p \Delta t p i A$ was much slower compared with that of the wild-type strain, and the final cell density was only $1.0 \pm 0.1 \mathrm{OD}$ unit. This strain needed 15 hours to consume all the glucose. 2,3-Butanediol and acetoin were still the main metabolites of this strain. But their titers were reduced to $2.1 \pm 0.5$ and $1.4 \pm 0.1 \mathrm{~g} / \mathrm{L}$, respectively. Neither DHA nor glycerol were detected in the fermentation broth. Thus, reversing the native glycerol catabolic pathway failed for DHA or glycerol production. 
As the DHA phosphate hydrolysis reaction has a high negative $\triangle \mathrm{rG}$ (No. 4 in Table 1). hdpA of $C$. glutamicum was heterologously expressed in $K p \Delta t p i A$ to construct $K p \Delta t p i A-h d p A$. This strain was cultured in flasks and results are shown in Fig. 2.

Cell growth of $K p \triangle t p i A-h d p A$ was slower than that of $K p \Delta t p i A$. The highest cell density was only $0.5 \pm 0.04 \mathrm{OD}$ units. Glucose was exhausted and $2.0 \pm 0.1 \mathrm{~g} / \mathrm{L}$ of 2,3 -butanediol and $1.5 \pm 0.1 \mathrm{~g} / \mathrm{L}$ of acetoin were produced after 21 hours of cultivation. DHA was produced in the process, and its concentration was continuously increasing, and a final tier of $7.0 \pm 0.3 \mathrm{~g} / \mathrm{L}$ was obtained. $2.5 \mathrm{~g} / \mathrm{L}$ of glycerol was also generated in the process. It should be pointed out that glycerol synthesis started later than DHA synthesis. Glycerol synthesis commenced after 15 hours of cultivation, at this time the DHA concentration reached $6.7 \mathrm{~g} / \mathrm{L}$. $K p$ hdpA, wild type strain with overexpression of $h d p A$, was also cultured. But cell growth and metabolite production of this strain were similar to those of the wild type strain. No DHA nor glycerol was produced by $K p$ hdpA (data not shown). In summary, a reverse glycerol catabolism pathway was set up based on the knock-out of tpiA and overexpression of $h d p A$, and DHA and glycerol were produced from glucose through this pathway.

\section{Blocking by-products synthesis in DHA production}

Metabolites of $K p \triangle t p i A-h d p A$ include DHA, glycerol, 2,3-butanediol and acetoin. If we consider DHA as the target product, then other metabolites are all by-products. $d h a D$ and $g / d A$ encoding isoenzymes of glycerol dehydrogenase that catalyse the conversion between DHA and glycerol. budA encoding acetolactate synthase, which is a key enzyme of 2,3-butanediol and acetoin synthesis pathway. Besides, DHA can be converted to methylglyoxal and further be converted to pyruvate (Fig. 1). Methylglyoxal synthase is encoded by $m g s A$. These key genes were knocked out individually or combined and pDK6hdpA was transformed to obtain $K p \Delta t p i A-\triangle m g s A-h d p A, K p \triangle t p i A-\triangle d h a D-h d p A, K p \triangle t p i A-\triangle m g s A-\Delta g / d A-$ $h d p A, K p \Delta t p i A-\triangle m g s A-\Delta g l d A-\triangle d h a D-h d p A$, and $K p \Delta t p i A-\triangle b u d A-h d p A$. These strains and the $K p \Delta t p i A-$ $h d p A$ were cultured in flasks and results are shown in Fig. 3.

Cell growth, glucose consumption, 2,3-butanediol and acetoin production of $K p \Delta t p i A-\Delta m g s A-h d p A$ were similar to those of $K p \Delta t p i A-h d p A .5 .6 \pm 0.1 \mathrm{~g} / \mathrm{L}$ of DHA and $2.5 \pm 0.1 \mathrm{~g} / \mathrm{L}$ of glycerol were produced by this strain. While, $6.4 \pm 0.5 \mathrm{~g} / \mathrm{L}$ of DHA and $2.2 \pm 0.2 \mathrm{~g} / \mathrm{L}$ of glycerol were produced by $K p \Delta t p i A-h d p A$. It indicated that blocking the pathway of DHA to methylglyoxal had no positive effect on DHA production.

Glycerol production by $K p \triangle t p i A-\triangle d h a D-h d p A$ was reduced, with the titer of $1.8 \pm 0.1 \mathrm{~g} / \mathrm{L}$. Whereas, no glycerol was produced by $K p \Delta t p i A-\Delta m g s A-\Delta g / d A-h d p A$. Kp $\Delta t p i A-\Delta m g s A-\Delta g / d A-\Delta d h a D-h d p A$ also produced no glycerol. However, DHA production by these three strains were all reduced remarkably, rather than increased. Cell growths of $K p \Delta t p i A-\Delta m g s A-\Delta g / d A-h d p A$ and $K p \triangle t p i A-\Delta m g s A-\Delta g / d A-\triangle d h a D-h d p A$ were enhanced, with final cell densities of $2.3 \pm 0.2$ and $2.1 \pm 0.1 \mathrm{OD}$ units, which were about four times that of $K p \triangle t p i A-h d p A$.

Neither 2,3-butanediol nor acetoin were synthesized by $K p \Delta t p i A-\triangle b u d A-h d p A$. Glucose consumption and cell growth of this strain became slow, and $6.5 \mathrm{~g} / \mathrm{L}$ of glucose was still unused in the broth after 24 hours 
of cultivation. Glycerol produced by this strain was similar to that of $K p \triangle t p i A-h d p A$. However, DHA titer of this strain was lower than that of strain $K p \Delta t p i A-h d p A$. Thus, blocking the 2,3-butanediol synthesis pathway had no positive effect on DHA and glycerol production.

These efforts to reduce glycerol or other by-products production all failed to enhance the level of DHA.

\section{Blocking the reaction of glyceraldehyde 3-phosphate to 1,3-bisphospho-glycerate for DHA production}

The knock-out of tpiA prevented further catabolism of DHA phosphate in the glycolysis and resulted in its conversion to DHA. Glyceraldehyde-3-phosphate dehydrogenase catalyses the conversion of glyceraldehyde 3-phosphate to 1,3-bisphospho-glycerate. It was suspected, that blocking further catabolism of glyceraldehyde 3-phosphate might also result in DHA phosphate flow to DHA. Glyceraldehyde-3-phosphate dehydrogenase has three isoenzymes as noted in the genome of Klebsiella variicola 342, and they are encoded by gap, gapA and gapC, respectively [19]. The genome of this strain was highly homologous to that of K.pneumoniae CGMCC 1.6366 used in this study. However, only gapA and gapC were found in the genome of K.pneumoniae CGMCC 1.6366. The two genes were knocked out individually and combined to get $K p \triangle g a p A, K p \Delta g a p C$ and $K p \Delta g a p A-\triangle g a p C$. pDK6-hdpA was transformed into these genes knock-out strains to get the corresponding strains. These strains were cultured in flasks and results were shown in Fig. 4.

Physiological characteristics of $K p \triangle g a p A$ were comparable to that of wild-type strain (data shown in Fig. 2). $23 \mathrm{~g} / \mathrm{L}$ of glucose was exhausted by these strains after 12 hours of cultivation. 2,3-Butanediol and acetoin were the main products of this strain, with the titer of $3.3 \pm 0.1$ and $2.5 \pm 0.3 \mathrm{~g} / \mathrm{L}$, respectively. Overexpression of $h d p A$ in $K p \triangle g a p A$ failed for DHA or glycerol production. $3.3 \pm 0.1 \mathrm{~g} / \mathrm{L}$ of 2,3-butanediol and $1.5 \pm 0.1 \mathrm{~g} / \mathrm{L}$ of acetoin were produced by $K p \triangle g a p A-h d p A$, which were similar to those of wild-type strain.

Cell growth of $K p \triangle g a p C$ was slightly slower compared to that of the wild-type strain, and glucose was exhausted after 15 hours of cultivation. 2,3-Butanediol and acetoin produced by this strain were similar to those of wild-type stain. Physiological characteristics of $K p \Delta g a p A-\triangle g a p C$ were nearly the same as those of $K p \triangle g a p C$.

Over-expression of $h d p A$ in $K p \Delta g a p C$ has a distinct effect on the host cell. Cell growth of $K p \Delta g a p C$-hdpA was very slow. After 33 hours of cultivation, $3 \mathrm{~g} / \mathrm{L}$ of glucose was still unused in the broth, and the cell density was lower than $1 \mathrm{OD}$ unit during most periods of the process.

2,3-Butanediol and acetoin produced by $K p \triangle g a p C$-hdpA were reduced to $1.4 \pm 0.1$ and $1.0 \pm 0.1 \mathrm{~g} / \mathrm{L}$, respectively. These metabolites were mainly synthesized in the beginning 6 hours of cultivation. $2.3 \pm 0.2$ $\mathrm{g} / \mathrm{L}$ of DHA was produced after 9 hours of cultivation, and after that its concentration had no distinct change. Glycerol was produced in the process, but its level was very low. $0.56 \mathrm{~g} / \mathrm{L}$ of glycerol was detected after 24 hours of cultivation. Metabolites of $K p \Delta g a p A-\triangle g a p C-h d p A$ were very likely that of $K p$ 
$\triangle g a p C-h d p A$. But the growth of this strain was very weak, and the highest cell density was only $0.2 \mathrm{OD}$ unit.

Blocking the conversion of glyceraldehyde 3-phosphate to 1,3-bisphospho-glycerate and over-expression of the $h p d A$ lead to DHA and glycerol production. However, the levels of DHA and glycerol produced by $K p$ $\triangle g a p C-h d p A$ or $K p \triangle g a p A-\triangle g a p C-h d p A$ were lower than those produced by $K p \triangle t p i A-h d p A$. This indicated that blocking further catabolism of DHA phosphate in the cell by knocking out of tpiA or gapC were both effective for DHA and glycerol production through the reverse glycerol catabolism pathway.

\section{The effect of disruption of DHA kinases on DHA production}

DHA kinases and DHA phosphate dephosphorylase catalyse the reaction of DHA phosphorylated and DHA phosphate dephosphorylated, respectively. If they both working at the same time, they form an futile cycle in the cell. To erase this reaction cycle, the subunits of the two kinases were disrupted individually and $h d p A$ was expressed to obtain the following strains: $K p \triangle t p i A-\triangle d h a K-h d p A, K p \triangle t p i A-\triangle d h a K 1-h d p A$, $K p \triangle t p i A-\triangle d h a K 2-h d p A, K p \triangle t p i A-\triangle d h a K 3-h d p A$, and $K p \triangle t p i A-\triangle D H A K-h d p A$. Kp $\triangle t p i A-\triangle D H A K-h d p A$ was a strain where $d h a K$, dhaK1, dhaK2 and dhaK3 were all knocked out. These strains were cultured in flasks and results are shown in Fig 5

Cell growth of $K p \Delta p t i A-\Delta d h a K-h d p A$ was slow compared with that of $K p \Delta p t i A-h d p A$. The final cell density of this strain was $0.3 \pm 0.1 \mathrm{OD}$ units. Accordingly, glucose consumption and metabolites productivity were all in low rates. $0.5 \pm 0.1 \mathrm{~g} / \mathrm{L}$ of 2,3-butanediol, $0.8 \pm 0.1 \mathrm{~g} / \mathrm{L}$ of acetoin and $3.6 \pm 0.5 \mathrm{~g} / \mathrm{L}$ of DHA were produced by this strain, while glycerol was not detected in the process.

Cell growth of $K p \triangle p t i A-\triangle d h a K 1-h d p A, K p \triangle p t i A-\triangle d h a K 2-h d p A$ and $K p \triangle p t i A-\triangle D H A K$-hdpA were faster than that of $K p \triangle p t i A-h d p A$. The highest cell densities of these strains were $0.8 \pm 0.1,0.6 \pm 0.1$ and $0.7 \pm 0.1$ $\mathrm{OD}$ units, respectively. Glucose consumption rate of these strains were faster than that of $K p \triangle p t i A-h d p A$. 2,3-Butanediol titers of these strains were $2.5 \pm 0.1,2.4 \pm 0.2$ and $2.3 \pm 0.1 \mathrm{~g} / \mathrm{L}$, respectively. Acetoin titer of these strains were similar to that of $K p \triangle p t i A-h d p A$. DHA produced by these strains were $7.2 \pm 0.4,7.0 \pm 0.2$ and $7.8 \pm 0.3 \mathrm{~g} / \mathrm{L}$, respectively. $K p \triangle p t i A-\triangle D H A K-h d p A$ had the highest DHA titer among these strains. Glycerol titers of these strains were similar to that of $K p \triangle p t i A-h d p A$, but productivities were higher than that of $K p \triangle p t i A-h d p A$. Cell growth and glucose consumption of $K p \triangle p t i A-\triangle d h a K 3-h d p A$ were similar to that of $K p \triangle p t i A-h d p A$. Glycerol produced by this strain was higher than $K p \triangle p t i A-h d p A$.

The DHA and glycerol production by these strains are summarized in Table 2.

Except $K p \triangle t p i A-\triangle d h a k-h d p A$, the conversion ratio of glucose to DHA and glycerol in these strains were all improved compared with that of $K p \triangle t p i A-h d p A$. The total conversion ratio of glucose to DHA and glycerol was 0.95 in $K p \triangle t p i A-\triangle D H A K-h d p A$, which was nearly the maximum theoretical conversion ratio. This strain also had the highest DHA and glycerol productivity and was selected for further investigation.

\section{DHA production by $K p \triangle t p i A-\triangle D H A K-h d p A$ using different carbon sources}


$K p \triangle t p i A-\triangle D H A K-h d p A$ was cultured in flasks with glucose, xylose, sucrose and fucose as the main carbon source, and results are shown in Fig. S1. All these carbon sources can be used by the cell for DHA and glycerol production. The conversion ratios of glucose, sucrose and fucose to DHA and glycerol were similar. The conversion ratio of xylose to DHA and glycerol was lower than others, with the value of 0.38 $\mathrm{mol} / \mathrm{mol}$. These results were reasonable. Sucrose was hydrolysed to form glucose and fructose, and the two monosaccharoses were all catabolised through the glycolysis pathway. Fucose is a monosaccharose that is rich in marine algae. In K. pneumoniae, fucose was converted to fuculose and further to fuculosephosphate. DHA-phosphate and lactaldehyde was formed from fuculose-phosphate with the catalysis of an aldolase. Xylose was catabolised through the Pentose phosphate pathway, only part of carbon was converted to DHA-phosphate and resulted a low conversion ratio to DHA and glycerol. While, more carbon was used for cell growth, and a high cell density was obtained with xylose as the carbon source. It can be concluded that any carbon source that can be catabolised to form DHA-phosphate is suitable for DHA and glycerol production by $K p \triangle t p i A-\triangle D H A K-h d p A$.

\section{Fermentation parameters optimization}

Culture $\mathrm{pH}$ optimization.

$K p \triangle t p i A-\triangle D H A K$ - $h d p A$ was cultured in $5 \mathrm{~L}$ bioreactors with fermentation medium, where the culture $\mathrm{pH}$ was stabilized at 5.5, 6.0,6.5, and 7.0. The air flow rate was set at $2 \mathrm{~L} / \mathrm{min}$ and the stirring rate of the bioreactor was set at 250 rpm. Fermentation results are presented in Fig. 6.

Cell growth was positively related to the culture $\mathrm{pH}$. The lowest and the highest cell densities were obtained at the culture pH 5.5 and 7.0, respectively. All cell densities obtained were higher than that in flask culture (shown in Fig. 5). Glucose consumption rates were similar in the range of pH 5.5-6.5. Glucose consumption rate in culture $\mathrm{pH} 7.0$ was low compared with other conditions, and it took 24 hours to use all the glucose supplied, which was slower than the consumption in flask culture.

2,3-Butanediol and acetoin production was inversely related to the culture $\mathrm{pH}$. $2.7 \mathrm{~g} / \mathrm{L}$ of 2,3-butanediol and $1.7 \mathrm{~g} / \mathrm{L}$ of acetoin were produced in culture $\mathrm{pH} 5.5$, while only $0.9 \mathrm{~g} / \mathrm{L}$ of 2,3-butanediol was produced in culture $\mathrm{pH} 7.0$, and no acetoin was detected at this condition.

DHA was produced from the beginning of cultivation, and quickly reached its high levels at 9 hours of cultivation. Glycerol production started at 9 hours, and its levels increased towards the end of the process. $6.1 \mathrm{~g} / \mathrm{L}$ of DHA was produced at culture $\mathrm{pH}$ 6.0. Lower or higher culture $\mathrm{pH}$ all resulted in lower DHA titers. The lowest DHA titer was obtained at culture $\mathrm{pH} 7.0$, reaching $4.1 \mathrm{~g} / \mathrm{L}$. Glycerol titers were in agreement with DHA titers. The highest glycerol titer was $3.1 \mathrm{~g} / \mathrm{L}$, which was obtained at culture $\mathrm{pH}$ 6.0.

DHA and glycerol titers and the substrate conversion ratios of these experiments are summarized in Table 3. The conversion ratio of glucose to $\mathrm{DHA}$ and glycerol both had the highest values at culture $\mathrm{pH} 6.0$, and the total value was 0.90 . Productivities of DHA and glycerol in culture $\mathrm{pH} 6.0$ were the fastest among all experimental conditions. Thus $\mathrm{pH} 6.0$ was selected as the optimized culture $\mathrm{pH}$. 
Oxygen supplementation

$K p \triangle t p i A-\triangle D H A K-h d p A$ was cultured in $5 \mathrm{~L}$ bioreactors. The air flow rate was set at $2 \mathrm{~L} / \mathrm{min}$ and the stirring rate of the bioreactor was set at 50,150,250, 250 and $450 \mathrm{rpm}$ to obtain different aerobic conditions. Culture $\mathrm{pH}$ were set at 6.0. Fermentation results are presented in Fig. 7.

Cells growth and glucose consumption had a positive relationship with stirring rates. The higher the stirring rate the higher the cell density and the glucose consumption rate. 3.6 OD unit was obtained at a stirring rate of $450 \mathrm{rpm}$. The lowest cell density was $2.3 \mathrm{OD}$ unit at stirring rate of $50 \mathrm{rpm}$. Glucose was exhausted after 13 hours of cultivation at a stirring rate of $450 \mathrm{rpm}$, followed by stirring rate of 350 and $250 \mathrm{rpm}$. Whilst 3.5 and $3.6 \mathrm{~g} / \mathrm{L}$ of glucose were still unused at stirring rate of 50 and $150 \mathrm{rpm}$ after 24 hours of cultivation. 2,3-Butanediol and acetoin productivities were improved with the increase of the stirring rate. Stirring rate of 50 and $150 \mathrm{rpm}$ had the lowest titers of 2,3-butanediol and acetoin.

The productivity of DHA at stirring rate of 50 and $150 \mathrm{rpm}$ conditions was similar, and these were the lowest value among all experimental conditions. However, the final titers of DHA were the highest, with the value of $7.1 \mathrm{~g} / \mathrm{L}$ and $7.4 \mathrm{~g} / \mathrm{L}$, respectively. At the same time, $3.3 \mathrm{~g} / \mathrm{L}$ and $3.1 \mathrm{~g} / \mathrm{L}$ of glycerol were produced, which were also the highest value among all agitation conditions.

The final titers of DHA and glycerol and substrate conversion ratios at different oxygen supplementation are summarized in Table 4. The total conversion ratio of glucose to DHA and glycerol was 0.97 and 0.98 at stirring rate of 50 and $150 \mathrm{rpm}$ conditions. It means nearly all DHA phosphate were transferred to DHA and glycerol in these conditions. In contrast, the total conversion ratio of glucose to DHA and glycerol was 0.58 at stirring rate of $550 \mathrm{rpm}$. Thus, lower oxygen supplementation favored DHA and glycerol production.

\section{Fed batch fermentation}

High culture $\mathrm{pH}$ and high oxygen supplementation favor for cell growth, but the conversion ratio of glucose to DHA and glycerol were low. Whereas low culture $\mathrm{pH}$ and low oxygen supplementation favor a high substrate conversion ratio, with low cell growth rate. To solve this inverse relationship, a two- phase culture strategy was used. In the beginning 6 hours of culture, the culture $\mathrm{pH}$ and stirring rate were set at 7.0 and $450 \mathrm{rpm}$, which were favorable for cell growth. Then, the culture $\mathrm{pH}$ and stirring rate were switched to 6.0 and $150 \mathrm{rpm}$, since this condition favored DHA and glycerol production. Fed batch fermentations were conducted to obtain a superior final product level. Fermentation results are presented in Fig. 8.

In fed batch fermentation, cells were rapidly growing during the first 16 hours of cultivation and the cell density reached to $2.1 \mathrm{OD}$ units. After about 30 hours cells entered a stationary phase and the highest cell density was reached at 39 hours of culture. The glucose concentration in the broth dropped to $2.4 \mathrm{~g} / \mathrm{L}$ after 26 hours. After addition of highly concentrated glucose solution due to this initial drop, the glucose concentration increased to $27.2 \mathrm{~g} / \mathrm{L}$. At 49 hours, another glucose feed had to be performed. DHA was 
produced in the broth and its concentration continued to increase. The highest DHA titer of $23.9 \mathrm{~g} / \mathrm{L}$ was achieved after 91 hours of cultivation. Glycerol production followed production of DHA and its titer was $10.8 \mathrm{~g} / \mathrm{L}$ after 91 hours of cultivation. The total conversion ratio of DHA and glycerol from glucose was $0.97 \mathrm{~mol} / \mathrm{mol}$. The main by-products of this process were 2,3-butanediol and acetoin, reaching a titer of 9.2 and $3.5 \mathrm{~g} / \mathrm{L}$, respectively.

DHA is a toxic chemical to the cell and high levels of DHA are known to inhibit the activity of cells [20]. The inhibitory effect of DHA on the wild-type K. pneumoniae was determined and results are shown in Fig. S2. As expected cell growth was inhibited by DHA. With the concentration of DHA in the broth increasing, the inhibitory effect became more obvious. Cell growth was totally stopped at $22 / \mathrm{L}$ of DHA. The $23.9 \mathrm{~g} / \mathrm{L}$ of DHA obtained in fed batch fermentation exceeded the highest level of DHA that cell can tolerate. Thus, cells might have lost metabolic activities in this condition; preventing the DHA titer to increase further.

\section{Discussion}

\section{Construction of a reverse glycerol catabolic pathway for DHA and glycerol production}

In our previous research about the isoenzymes of DHA kinase, it was found that disruption of DHA kinase I had no effect on glycerol catabolism. Whereas the activity of DHA kinase II was essential for glycerol catabolism [8]. This agrees with the thermodynamic analysis of the glycerol catabolic pathway shown in Table 1. DHA kinase II should be the functional enzyme in glycerol catabolism as the reaction of glycerol convertion to DHA that is catalysed by DhaD prefers the reverse direction. The glycerol catabolism pathway was favored by the high negative $\triangle \mathrm{rG}$ of the reaction of DHA phosphorylation. Thus, DHA concentration in the cell was at a very low level and could not accumulate in the broth. An Saccharomyces cerevisiae strain was constructed for DHA production, and a NAD-dependent glycerol dehydrogenase was used for DHA formation from glycerol. This strain produced very low level of DHA with the titer of $0.7 \mathrm{~g} / \mathrm{L}$ [21]. The reaction of glycerol to DHA combined with NADH formation has the same $\Delta r G$, regardless of different glycerol dehydrogenase used. Thus, it was not possible to achieve high level of DHA production using NADH-linked glycerol dehydrogenase. However, DHA could be produced from glycerol by the cells of $G$. oxydans [12]. The enzyme catalysis this reaction was a membrane-bound enzyme and located in the periplasmic space. This glycerol dehydrogenase uses PQQ instead of NAD as the cofactor. The $\triangle \mathrm{rG}$ of this reaction was negative, and the reaction equilibrium favors DHA formation.

Glucose is a commonly used carbon source for bacterial growth. DHA phosphate is an intermediate of the glycolysis. Neither the wild-type K. pneumoniae nor the $K p \triangle t p i A$ produce DHA or glycerol (Fig. 2), it indicated that the native glycerol catabolism pathway was difficult to work in the reverse direction. $h d p A$ encode a haloacid dehalogenase superfamily member that catalyzes dephosphorylation of DHA phosphate to produce DHA in C. glutamicum [22]. After $h d p A$ was introduced to the cell, a considerable level of DHA and glycerol was produced by $K p \Delta t p i A-h d p A$ (Fig 2). This was reasonable, because the $\Delta \mathrm{rG}$ of this reverse glycerol catabolism pathway was estimated to be $-37.3 \mathrm{~kJ} / \mathrm{mol}$ (Table 1). Besides 
overexpression of $h d p A$, the disruption of tpiA was also essential for DHA and glycerol production. In the research of 1,3-propanediol production from glucose and use $E$. coli as the host cell the inactivation of tpiA lead to assembling the carbon pathway from DHA phosphate to 1,3-propanediol [23]. In this research DHA phosphate was first reduced to glycerol phosphate and the latter was converted to glycerol, glycerol3-phosphate dehydrogenase and glycerol-3-phosphatase cloned from Saccharomyces cerevisiae were used to catalyse the reaction. Glycerol was synthesized in a similar way when $K$. pneumoniae was used as the host [24].

\section{GIdA, rather than DhaD is the main enzyme that catalyses glycerol formation from DHA}

GldA and DhaD are isoenzymes of glycerol dehydrogenase. The amino acid sequence of the two enzymes exhibits high identities, but their expression was different. $d h a D$ is a gene in the dha operon. It was reported that the expression of the dha operon was induced by DHA [9], and DhaR and DhaK123 were involved in the induction process [8]. $g / d A$ is an independent gene in the genome of $K$. pneumoniae, and there are no regulatory genes nearby. It has been noted that the expression of $d h a D$ and $g / d A$ were both at low levels when cells were cultured on glucose as carbon source [25]. The expression of $d h a D$ was induced by glycerol but the expression of $g / d A$ was not subject to glycerol induction [25]. DHA and glycerol were produced by $K p \triangle t p i A-h d p A$ with glucose as the carbon source. The results shown in Fig. 3 indicated GldA was the main functional enzyme catalysing the formation of glycerol. It indicated that the expression of $d h a D$ was inhibited by glucose, even glycerol and DHA were present in the broth.

Glycerol was also produced in a report on DHA production from glucose by engineering $E$. coli. In that work the glycerol titer was reduced and DHA titer was increased by knocking out gldA [16]. This was different to the results obtained in this study. Glycerol production was reduced by the strains $K p \triangle t p i A$ $\triangle m g s A-\Delta g l d A-h d p A$ and $K p \Delta t p i A-\triangle d h a D-h d p A$. However, DHA production was also reduced by these strains.

\section{DHA production by blocking the conversion of glyceraldehyde-3-phosphate to 1,3-bisphospho-glycerate}

gapA and gapC encode isoenzymes of glyceraldehyde-3-phosphate dehydrogenase. Physiological characteristics of $K p \Delta g a p A, K p \Delta g a p C$ and $K p \triangle g a p C-\Delta g a p A$ were like that of the wild-type strain. This indicated, other isoenzymes or pathways might exist in the cell that are responsible for glyceraldehyde-3phosphate catabolism. DHA and glycerol were produced by $K p \triangle g a p C-h d p A$ and $K p \triangle g a p C-\triangle g a p A-h d p A$, but not by $K p \triangle g a p A-h d p A$. This indicated, the isoenzyme of glyceraldehyde-3-phosphate dehydrogenase that is encoded by $g a p C$ was the principal functionalal enzyme in the cell. Inactivation of GapC blocked or partly blocked the further metabolism of glyceraldehyde-3-phosphate. The strategy of down-regulation of glyceraldehyde-3-phosphate dehydrogenase was done to assemble the carbon pathway from DHA phosphate to 1,3-propanediol, and this strategy was better than the knockout of tpiA in terms of 1,3propanediol yield [23]. It was different to the DHA and glycerol production in this study, where the knockout of tpiA resulted in a higher yield of DHA and glycerol.

\section{Roles of DHA kinases on DHA production}


DHA kinase I and DHA kinase II are isoenzymes, that catalyse the formation of DHA phosphate from DHA. Beside the catalysis function, subunits of DHA kinase II are involved in the regulation of the $d h a$ operon [8]. The DHA produced by $K p \triangle p t i A-\triangle d h a K 1-h d p A, K p \triangle p t i A-\triangle d h a K 2-h d p A, K p \triangle p t i A-\triangle d h a K 3-h d p A$ and $K p \triangle t p i A-\triangle D H A K-h d p A$ were increased compared with that of $K p \triangle t p i A-h d p A$ indicating that DHA kinase II was active in $K p \triangle t p i A-h d p A$. Whereas knocking out $d$ haK had no effect on glycerol catabolism [8] and cell growth and DHA production by $K p \triangle p t i A-\Delta d h a K$-hdpA was decreased compared with $K p$ $\triangle t p i A-h d p A$. The function of DHA kinase I in DHA and glycerol production from glucose remains unclear.

\section{DHA production by $K p \triangle t p i A-\triangle D H A K-h d p A$}

In the flasks culture of $K p \triangle t p i A-\triangle D H A K-h d p A$, a total substrate conversion ratio of 0.95 was obtained. It indicated nearly all the glucose was catabolised through the glycolysis pathway, and the DHA phosphate formed in the glycolysis was converted to DHA. Due to the lack of $\mathrm{pH}$ control in the flask the $\mathrm{pH}$ continuously decreased during the cultivation process. It was confirmed in the stirred tank bioreactors experiments that a low pH is favorable for DHA and glycerol production. $\mathrm{pH} 6.0$ had the highest DHA and glycerol titers, but DHA and glycerol production at $\mathrm{pH} 5.5$ were low. The likely reason was some gluconic acid and 2-ketogluconic acid were formed through the glucose oxidization pathway that is located in the periplasmic space, and acidic condition favor gluconic acid and 2-ketogluconic acid accumulation [1, 2]. At neutral $\mathrm{pH}$ conditions or high oxygen supplement conditions, cell growth of $K p \Delta t p i A-\triangle D H A K-h d p A$ were enhanced, but all metabolites were decreased. Instead of DHA phosphate, 3-phosphoglyceraldehyde and pyruvate were formed as intermediate of the glucose oxidization pathway [1]. Glucose catabolism through oxidization pathway rather than glycolysis will reduce the formation of DHA phosphate, and leads to low conversion ratio of glucose to DHA and glycerol.

In this study, $7.8 \mathrm{~g} / \mathrm{L}$ of DHA and $1.9 \mathrm{~g} / \mathrm{L}$ of glycerol were produced by $K p \triangle t p i A-\triangle D H A K$ - $h d p A$ after 15 hours of cultivation in flasks. The final titer, productivity and substrate conversion ratio obtained in this study were all higher than that in the report of DHA production by engineering $E$. coli. In that work $6.6 \mathrm{~g} / \mathrm{l}$ of DHA was produced after 40 hours of cultivation, and the substrate conversion ratio was 0.87 [16]. 23.9 $\mathrm{g} / \mathrm{L}$ of DHA and $10.8 \mathrm{~g} / \mathrm{L}$ of glycerol obtained in fed batch fermentation provides the basis for a novel and highly efficient way of DHA and glycerol production from glucose.

\section{Methods}

\section{Strains, plasmids, and primers}

Bacterial strains and plasmids used in this study are listed in Table 5. Primers used for PCR are listed in Table S1.

\section{Gibbs free energy reaction estimation}

Gibbs free energy of metabolic reactions were estimated with the help of the eQuilibrator (http://equilibrator.weizmann.ac.il). eQuilibrator is a simple web interface designed to enable easy 
thermodynamic analysis of biochemical systems. All the substrate and cofactor were set at standard conditions with the concentration of $1 \mathrm{~mol} / \mathrm{L}, \mathrm{pH} 7.0$ and ionic strength $0.1 \mathrm{~mol} / \mathrm{L}$.

\section{Knocking out genes in the genome of $K$. pneumoniae}

For genes knock out, K. pneumoniae and E. coli were grown in Luria-Bertani (LB) medium at $37^{\circ} \mathrm{C}$. The antibiotics used in the selective medium were ampicillin $(50 \mu \mathrm{g} / \mathrm{mL})$, kanamycin $(50 \mu \mathrm{g} / \mathrm{mL})$, apramycin $(50 \mu \mathrm{g} / \mathrm{mL})$, and streptomycin $(25 \mu \mathrm{g} / \mathrm{mL})$.

\section{$K p \Delta t p i A$ construction}

$K p \triangle t p i A$ and other gene knock out strains constructions were generated using the Red recombinase associated gene replacement method as described previously with some modification [28]. The up and drown flanking sequences of tpiA gene in the genome of $K$. pneumoniae were amplified by PCR using the primer pair tpiA-up-s/a and ptiA-down-s/a. Apramycin resistance gene aac(3)IV was amplified with plasmid pIJ773 as the template using the primer pair tpiA-FRT-s/a. The up and drown flanking sequences of tpiA and $\operatorname{aac}(3) I V$ were ligated together with a ClonExpress Ultra One Step Cloning Kit@ to generate a linear DNA containing the apramycin resistance gene aac(3)IV with $600 \mathrm{bp}$ of tpiA homologous regions on both sides. The linear DNA was transformed into K. pneumoniae CGMCC 1.6366, which already hosted the plasmid pDK6-red. Homologous recombination between the linear DNA and the chromosome was facilitated by Red recombinase and led to tpiA deletion in the genome of $K$. pneumoniae to obtain $K p$ $\Delta t p i$.

\section{K. pneumoniae $\Delta$ tpi- $\Delta$ dhak construction}

$K p \Delta t p i-\Delta d h a k$ was constructed following the same way of $K p \Delta t p i$ construction, with $K p \Delta d h a k$ [8] replacing wild-type $K$. pneumoniae as the target strain.

The ORF of $h d p A$ in C. glutamicum was amplified using the primer pair $h d p A-s$ and $h d p A-s$. The PCR product was digested with restriction enzyme EcoR I and BamH I. The DNA fragment was ligated into the pDK6 vector to generate pDK6-hdpA. pDK6-hdpA was transformed into $K p \triangle t p i$ to generate $K p \Delta t p i-h d p A$. Other strains with expression of $h d p A$ were constructed in the same way as $K p \Delta t p i-h d p A$.

\section{Medium and culture conditions}

The fermentation medium contained $25 \mathrm{~g} / \mathrm{L}$ glucose, $1.5 \mathrm{~g} / \mathrm{L}$ yeast extract, $4 \mathrm{~g} / \mathrm{L}\left(\mathrm{NH}_{4}\right)_{2} \mathrm{SO}_{4}, 0.69 \mathrm{~g} / \mathrm{L}$ $\mathrm{K}_{2} \mathrm{HPO}_{4} \cdot 3 \mathrm{H}_{2} \mathrm{O}, 0.25 \mathrm{~g} / \mathrm{L} \mathrm{KH}_{2} \mathrm{PO}_{4}, 0.2 \mathrm{~g} / \mathrm{L} \mathrm{MgSO}_{4}, 0.05 \mathrm{~g} / \mathrm{L} \mathrm{FeSO}_{4} \cdot 7 \mathrm{H}_{2} \mathrm{O}$ and $1 \mathrm{~mL}$ of trace element solution. One liter of trace element solution contained $200 \mathrm{mg} \mathrm{CoCl} 2 \cdot 6 \mathrm{H}_{2} \mathrm{O}, 100 \mathrm{mg} \mathrm{MnSO}{ }_{4} \cdot 4 \mathrm{H}_{2} \mathrm{O}, 70 \mathrm{mg}$ $\mathrm{ZnCl}_{2}, 60 \mathrm{mg} \mathrm{H}_{3} \mathrm{BO}_{3}, 35 \mathrm{mg} \mathrm{Na}_{2} \mathrm{MoO}_{4} \cdot 2 \mathrm{H}_{2} \mathrm{O}, 29.28 \mathrm{mg} \mathrm{CuSO}{ }_{4} \cdot 5 \mathrm{H}_{2} \mathrm{O}, 25 \mathrm{mg} \mathrm{NiCl} \cdot 6 \mathrm{H}_{2} \mathrm{O}$ and $0.9 \mathrm{~mL} 37 \%$ $\mathrm{HCl}$.

K. pneumoniae strains were inoculated in $250 \mathrm{ml}$ flasks containing $50 \mathrm{ml}$ medium and incubated on a rotary shaker at $37^{\circ} \mathrm{C}$ and $120 \mathrm{rpm}$ for 1 day. All experiments were done in triplicate, and data are 
expressed as the mean \pm standard error.

The inhibitory effect of DHA on cell growth was determined in flask culture. Wild type K. pneumoniae were inoculated in $250 \mathrm{ml}$ flasks containing $50 \mathrm{ml} \mathrm{LB}$ medium, and DHA was added in the medium to a obtain a range of different concentrations.

Fermentation parameters such as culture $\mathrm{pH}$ and oxygen supplementation optimization experiments were performed in batch bioreactor fermentation systems. For the seed culture, $250 \mathrm{~mL}$ flasks containing 50 $\mathrm{mL}$ of LB medium were incubated in a rotary shaker at $37^{\circ} \mathrm{C}$ and $200 \mathrm{rpm}$ overnight. Seed culture was inoculated into 5-L tank bioreactor (BIOSTAT-A plus, Sartorius) with a working volume of $3 \mathrm{~L}$ and the air flow rate was $2 \mathrm{~L} / \mathrm{min}$. The culture $\mathrm{pH}$ was automatically controlled by $10 \mathrm{~mol} / \mathrm{L} \mathrm{NaOH}$ addition. For fed batch culture, glucose was fed when its concentration in the broth was dropped to $5 \mathrm{~g} / \mathrm{L}$.

\section{Analytical methods}

The biomass yield at set time intervals was determined by optical density (OD600) with a spectrophotometer. Chemical compounds in the broth were quantified by a Shimadzu 20AVP high performance liquid chromatography system (HPLC) equipped with a RID-20A refractive index detector. An Aminex HPX-87H column $(300 \times 7.8 \mathrm{~mm})$ (Bio-Rad, USA) was used and the column temperature was set at $65^{\circ} \mathrm{C}$. The mobile phase was $0.005 \mathrm{~mol} / \mathrm{L} \mathrm{H}_{2} \mathrm{SO}_{4}$ solution with a flow rate of $0.8 \mathrm{ml} / \mathrm{min}$.

The retention times of glycerol and DHA for HPLC were the same, thus gas chromatography (GC) was used to detect the levels of glycerol and DHA. A gas chromatography system (Shimadzu GC 2010) equipped with a flame ionization detector and a HP-FFAP column $(30 \mathrm{~m} \times 0.25 \mathrm{~mm})$, with nitrogen as the carrier gas was used. The injector and detector were maintained at $250^{\circ} \mathrm{C}$ and $280^{\circ} \mathrm{C}$, respectively. The column temperature initially started at $120^{\circ} \mathrm{C}$ and was maintained for $1 \mathrm{~min}$, then increased to $230^{\circ} \mathrm{C}$ at a rate of $20^{\circ} \mathrm{C} / \mathrm{min}$, then maintained at this temperature for $1.3 \mathrm{~min}$.

\section{Declarations}

\section{Ethics approval and consent to participate}

Not applicable

\section{Consent for publication}

Not applicable

\section{Conflict of Interest}

Authors declare that they have no conflict of interest.

\section{Funding}


This work was supported by National Key R\&D Program of China (Grant No. 2019YFE0196900, 2017YFE0112700), Natural Science Foundation of Shanghai (Grant No. 19ZR1463600) , Royal SocietyNewton Advanced Fellowship (Grant No. NAF\R2\180721).

\section{Authors' contributions}

FB and JH designed this study. SS, YY, LS, $X L$ and $Q W$ conducted the research. SS, YY, CZ, JS, GL, FB and $\mathrm{JH}$ analysed the data. SS, FB and $\mathrm{JH}$ wrote the manuscript. All authors read and approved the final manuscript.

Acknowledgments FB would like to thank the Chinese Academy of Sciences for the award of a President's International Fellowship Initiative (Grant No. 2019VCB0007).

\section{References}

1. Wei D, Xu J, Sun J, Shi J, Hao J: 2-Ketogluconic acid production by Klebsiella pneumoniae CGMCC 1.6366. J Ind Microbiol Biotechnol 2013, 40:561-570.

2. Wang D, Wang C, Wei D, Shi J, Kim CH, Jiang B, Han Z, Hao J: Gluconic acid production by gad mutant of Klebsiella pneumoniae. World J Microbiol Biotechnol 2016, 32:132.

3. Wang C, Wei D, Zhang Z, Wang D, Shi J, Kim CH, Jiang B, Han Z, Hao J: Production of xylonic acid by Klebsiella pneumoniae. Appl Microbiol Biotechnol 2016, 100:10055-10063.

4. Wang D, Zhou J, Chen C, Wei D, Shi J, Jiang B, Liu P, Hao J: R-acetoin accumulation and dissimilation in Klebsiella pneumoniae. J Ind Microbiol Biotechnol 2015, 42:1105-1115.

5. Wang Y, Gu J, Lu X, Zhang Z, Yang Y, Sun S, Kostas ET, Shi J, Gao M, Baganz F, et al: 2,3Dihydroxyisovalerate production by Klebsiella pneumoniae. Appl Microbiol Biotechnol 2020, 104:6601-6613.

6. Gu J, Zhou J, Zhang Z, Kim CH, Jiang B, Shi J, Hao J: Isobutanol and 2-ketoisovalerate production by Klebsiella pneumoniae via a native pathway. Metab Eng 2017, 43:71-84.

7. Hao J, Lin R, Zheng Z, Liu H, Liu D: Isolation and characterization of microorganisms able to produce 1, 3-propanediol under aerobic conditions. World J Microbiol Biotechnol 2008, 24:1731-1740.

8. Wei D, Wang M, Jiang B, Shi J, Hao J: Role of dihydroxyacetone kinases I and II in the dha regulon of Klebsiella pneumoniae. J Biotechnol 2014, 177:13-19.

9. Forage RG, Lin EC: DHA system mediating aerobic and anaerobic dissimilation of glycerol in Klebsiella pneumoniae NCIB 418. J Bacteriol 1982, 151:591-599.

10. Mishra R, Jain S, Kumar A: Microbial production of dihydroxyacetone. Biotechnol Adv 2008, 26:293303.

11. Castells J, Geijo F, López-Calahorra F: The "formoin reaction" : A promising entry to carbohydrates from formaldehyde. Tetrahedron Lett 1980, 21:4517-4520. 
12. Gätgens C, Degner U, Bringer-Meyer S, Herrmann U: Biotransformation of glycerol to dihydroxyacetone by recombinant Gluconobacter oxydans DSM 2343. Appl Microbiol Biotechnol $2007,76: 553-559$.

13. Liu Z, Hu Z, Zheng Y, Shen Y: Optimization of cultivation conditions for the production of 1,3dihydroxyacetone by Pichia membranifaciens using response surface methodology. Biochem Eng $\mathrm{J}$ 2008, 38:285-291.

14. Kato N, Kobayashi H, Shimao M, Sakazawa C: Dihydroxyacetone production from methanol by a dihydroxyacetone kinase deficient mutant of Hansenula polymorpha. Appl Microbiol Biotechnol 1986, 23:180-186.

15. Siebert D, Wendisch VF: Metabolic pathway engineering for production of 1,2-propanediol and 1propanol by Corynebacterium glutamicum. Biotechnol Biofuels 2015, 8:91.

16. Jain VK, Tear CJY, Lim CY: Dihydroxyacetone production in an engineered Escherichia colithrough expression of Corynebacterium glutamicum dihydroxyacetone phosphate dephosphorylase. Enzyme Microb Technol 2016, 86:39-44.

17. Chen C, Wei D, Shi J, Wang M, Hao J: Mechanism of 2, 3-butanediol stereoisomer formation in Klebsiella pneumoniae. Appl Microbiol Biotechnol 2014, 98:4603-4613.

18. Upchurch RG, Mortenson LE: In vivo energetics and control of nitrogen fixation: changes in the adenylate energy charge and adenosine 5 '-diphosphate/adenosine 5 '-triphosphate ratio of cells during growth on dinitrogen versus growth on ammonia. J Bacteriol 1980, 143:274-284.

19. Fouts DE, Tyler HL, DeBoy RT, Daugherty S, Ren Q, Badger JH, Durkin AS, Huot H, Shrivastava S, Kothari S, et al: Complete genome sequence of the N2-fixing broad host range endophyte Klebsiella pneumoniae 342 and virulence predictions verified in mice. PLoS Genet 2008, 4:e1000141.

20. Wang L, Chauliac D, Rhee MS, Panneerselvam A, Ingram LO, Shanmugam KT: Fermentation of dihydroxyacetone by engineered Escherichia coli and Klebsiella variicola to products. Proc Natl Acad Sci USA 2018, 115:4381-4386.

21. Nguyen H, Nevoigt E: Engineering of Saccharomyces cerevisiae for the production of dihydroxyacetone (DHA) from sugars: A proof of concept. Metab Eng 2009.

22. Jojima T, Igari T, Gunji W, Suda M, Inui M, Yukawa H: Identification of a HAD superfamily phosphatase, HdpA, involved in 1,3-dihydroxyacetone production during sugar catabolism in Corynebacterium glutamicum. FEBS Lett 2012, 586:4228-4232.

23. Nakamura $\mathrm{CE}$, Whited GM: Metabolic engineering for the microbial production of 1,3-propanediol. Curr Opin Biotechnol 2003, 14:454-459.

24. Zheng Y, Zhao L, Zhang J, Zhang H, Ma X, Wei D: Production of glycerol from glucose by coexpressing glycerol-3-phosphate dehydrogenase and glycerol-3-phosphatase in Klebsiella pneumoniae. J Biosci Bioeng 2008, 105:508-512.

25. Wang Y, Tao F, Xu P: Glycerol dehydrogenase plays a dual role in glycerol metabolism and 2,3butanediol formation in Klebsiella pneumoniae. J Biol Chem 2014, 289:6080-6090. 
26. Gust B, Challis GL, Fowler K, Kieser T, Chater KF: PCR-targeted Streptomyces gene replacement identifies a protein domain needed for biosynthesis of the sesquiterpene soil odor geosmin. Proc Natl Acad Sci USA 2003, 100:1541-1546.

27. Kleiner D, Paul W, Merrick MJ: 1988. Construction of multicopy expression vectors for regulated overproduction of proteins in Klebsiella pneumoniae and other enteric bacteria. Microbiol 134: 17791784.

28. Wei $\mathrm{D}$, Wang $\mathrm{M}$, Shi J, Hao J: Red recombinase assisted gene replacement in Klebsiella pneumoniae. J Ind Microbiol Biotechnol 2012, 39:1219-1226.

\section{Tables}

Table 1. Gibbs free energy for reactions

\begin{tabular}{|c|c|c|c|c|}
\hline No. & Reactions and pathways & Enzyme & $\begin{array}{l}\Delta \mathrm{rG} \\
(\mathrm{kJ} / \mathrm{mol})\end{array}$ & $\mathrm{K}_{\mathrm{eq}}$ \\
\hline 1 & Glycerol $+\mathrm{NAD}^{+}=\mathrm{DHA}+\mathrm{NADH}+\mathrm{H}^{+}$ & DhaD & $24.6 \pm 3.6$ & $\begin{array}{l}4.9 \times 10 \\
5\end{array}$ \\
\hline 2 & $D H A+A T P=D H A-P+A D P$ & Dhak & $-13.7 \pm 4.3$ & 255 \\
\hline 3 & $\mathrm{DHA}+\mathrm{PEP}=\mathrm{DHA}-\mathrm{P}+$ pyruvate & Dhak123 & $-41.4 \pm 4.4$ & $\begin{array}{l}1.8 \times \\
10^{7}\end{array}$ \\
\hline 4 & DHA-P $+\mathrm{H}_{2} \mathrm{O}=\mathrm{DHA}+$ phosphate & HdpA & $-12.7 \pm 4.3$ & 166 \\
\hline $1+2$ & ATP $+\mathrm{NAD}^{+}+$Glycerol $=\mathrm{NADH}+\mathrm{ADP}+\mathrm{DHA}-\mathrm{P}$ & DhaD, Dhak & 10.9 & \\
\hline $1+3$ & $\begin{array}{l}\text { PEP }+\mathrm{NAD}^{+}+\text {Glycerol }=\mathrm{NADH}+\text { pyruvate }+ \\
\text { DHA-P }\end{array}$ & $\begin{array}{l}\text { DhaD, } \\
\text { Dhak123 }\end{array}$ & -16.8 & \\
\hline $4-1$ & $\mathrm{NADH}+\mathrm{DHA}-\mathrm{P}=\mathrm{NAD}{ }^{+}+$Glycerol + phosphate & DhaD, HdpA & -37.3 & \\
\hline
\end{tabular}

Table 2. DHA and glycerol production by strains of inactive isoenzymes of DHA kinase 


\begin{tabular}{|c|c|c|c|c|c|c|c|}
\hline Strains & $\begin{array}{l}\text { Time } \\
\text { (h) }\end{array}$ & $\begin{array}{l}\text { Glucose } \\
\text { consumed } \\
(\mathrm{g} / \mathrm{L})\end{array}$ & $\begin{array}{l}\text { DHA } \\
(\mathrm{g} / \mathrm{L})\end{array}$ & $\begin{array}{l}\text { Glycerol } \\
(\mathrm{g} / \mathrm{L})\end{array}$ & $\begin{array}{l}\text { DHA } \\
\text { conversion } \\
\text { ratio (mol/ } \\
\text { mol) }\end{array}$ & $\begin{array}{l}\text { Glycerol } \\
\text { conversion } \\
\text { ratio (mol/ } \\
\text { mol) }\end{array}$ & $\begin{array}{l}\text { Total } \\
\text { conversion } \\
\text { ratio (mol/ } \\
\text { mol) }\end{array}$ \\
\hline $\begin{array}{l}\Delta \text { tpiA- } \\
\text { hdpA }\end{array}$ & 18 & 22.6 & 6.7 & 1.9 & 0.60 & 0.17 & 0.76 \\
\hline $\begin{array}{l}\Delta \text { tpiA- } \\
\Delta \text { dhak- } \\
\text { hdpA }\end{array}$ & 24 & 15.4 & 3.1 & 0 & 0.41 & 0 & 0.41 \\
\hline $\begin{array}{l}\Delta \text { tpiA- } \\
\Delta \text { dhak1- } \\
\text { hdpA }\end{array}$ & 15 & 22.4 & 7.2 & 2.0 & 0.65 & 0.18 & 0.83 \\
\hline $\begin{array}{l}\Delta \text { tpiA- } \\
\Delta \text { dhak2- } \\
\text { hdpA }\end{array}$ & 15 & 20.4 & 7.0 & 2.1 & 0.69 & 0.20 & 0.89 \\
\hline $\begin{array}{l}\Delta \text { tpiA- } \\
\Delta \text { dhak3- } \\
\text { hdpA }\end{array}$ & 18 & 20.0 & 6.8 & 2.1 & 0.68 & 0.21 & 0.88 \\
\hline $\begin{array}{l}\Delta \text { tpiA- } \\
\triangle \mathrm{DHAK}- \\
\mathrm{hdpA}\end{array}$ & 15 & 20.4 & 7.8 & 1.9 & 0.76 & 0.19 & 0.95 \\
\hline
\end{tabular}

Table 3. DHA and glycerol production by $K p \triangle t p i A-\triangle D H A K-h d p A$ at different culture $\mathrm{pH}$.

\begin{tabular}{|llllllll|}
\hline $\begin{array}{l}\text { Culture } \\
\mathrm{pH}\end{array}$ & $\begin{array}{l}\text { Time } \\
(\mathrm{h})\end{array}$ & $\begin{array}{l}\text { Glucose } \\
\text { consumed } \\
(\mathrm{g} / \mathrm{L})\end{array}$ & $\begin{array}{l}\text { DHA } \\
(\mathrm{g} / \mathrm{L})\end{array}$ & $\begin{array}{l}\text { Glycerol } \\
(\mathrm{g} / \mathrm{L})\end{array}$ & $\begin{array}{l}\text { DHA } \\
\text { conversion } \\
\text { ratio }(\mathrm{mol} / \\
\mathrm{mol})\end{array}$ & $\begin{array}{l}\text { Glycerol } \\
\text { conversion } \\
\text { ratio }(\mathrm{mol} / \\
\mathrm{mol})\end{array}$ & $\begin{array}{l}\text { Sum } \\
\text { conversion } \\
\text { ratio }(\mathrm{mol} / \\
\mathrm{mol})\end{array}$ \\
\hline 5.5 & 18 & 22.2 & 5.4 & 2.1 & 0.49 & 0.19 & 0.68 \\
\hline 6.0 & 18 & 20.4 & 6.1 & 3.1 & 0.60 & 0.30 & 0.90 \\
\hline 6.5 & 18 & 20.5 & 4.6 & 3.1 & 0.45 & 0.30 & 0.75 \\
\hline 7.0 & 21 & 21.5 & 3.8 & 2.3 & 0.35 & 0.21 & 0.57 \\
\hline
\end{tabular}

Table 4. DHA and glycerol production by $K p \triangle t p i A-\triangle D H A K-h d p A$ at different stirring rates. 


\begin{tabular}{|llllllll|}
\hline $\begin{array}{l}\text { Stirring } \\
\text { rate }\end{array}$ & $\begin{array}{l}\text { Time } \\
(\mathrm{h})\end{array}$ & $\begin{array}{l}\text { Glucose } \\
\text { consumed } \\
(\mathrm{g} / \mathrm{L})\end{array}$ & $\begin{array}{l}\text { DHA } \\
(\mathrm{g} / \mathrm{L})\end{array}$ & $\begin{array}{l}\text { Glycerol } \\
(\mathrm{g} / \mathrm{L})\end{array}$ & $\begin{array}{l}\text { DHA } \\
\text { conversion } \\
\text { ratio }(\mathrm{mol} / \\
\mathrm{mol})\end{array}$ & $\begin{array}{l}\text { Glycerol } \\
\text { conversion } \\
\text { ratio (mol/ } \\
\mathrm{mol})\end{array}$ & $\begin{array}{l}\text { Total } \\
\text { conversion } \\
\text { ratio }(\mathrm{mol} / \\
\mathrm{mol})\end{array}$ \\
\hline 50 & 24 & 21.1 & 7.1 & 3.2 & 0.67 & 0.30 & 0.97 \\
\hline 150 & 24 & 21.4 & 7.4 & 3.1 & 0.69 & 0.29 & 0.98 \\
\hline 250 & 21 & 20.4 & 6.1 & 3.1 & 0.60 & 0.30 & 0.90 \\
\hline 350 & 15 & 23.9 & 6.4 & 2.0 & 0.54 & 0.17 & 0.70 \\
\hline 450 & 13 & 23.2 & 5.3 & 1.6 & 0.44 & 0.13 & 0.58 \\
\hline
\end{tabular}

Table 5 Strains and plasmids 
Strains or plasmids

Corynebacterium glutamicum

ATCC 13032

E.coli DH5a

K.pneumoniae CGMCC 1.6366

$\Delta$ tpiA

$\Delta$ tpiA- $\Delta$ mgsA

$\Delta$ tpiA- $\Delta$ mgsA- $\Delta$ gldA

$\Delta$ tpiA- $\Delta$ mgsA- $\Delta$ gldA- $\Delta$ dhaD

$\Delta$ tpiA- $\Delta$ dhaD

$\Delta$ tpiA- $\Delta$ budA

$\Delta$ gapA

$\Delta \mathrm{gapC}$

$\Delta$ gapA- $\Delta$ gapC

$\Delta$ tpiA- $\Delta$ dhaK

$\Delta$ tpiA- $\Delta$ dhaK1

$\Delta$ tpiA- $\Delta$ dhaK2

$\Delta$ tpiA- $\Delta$ dhaK3

$\triangle$ tpiA- $\triangle$ DHAK

Plasmids

plJ773

plJ778

pDK6

pDK6-hdpA

pDK6-red

pDK6-flp
Relevant genotype and description

Host of plasmid

TUAC01 Wild type, Amp ${ }^{r}$

$\Delta$ tpiA, Apr ${ }^{r}$

$\Delta$ mgsA, $\Delta$ tpiA, Apr ${ }^{r}, \operatorname{Str}^{r}$

$\Delta \mathrm{mgsA}, \Delta \mathrm{tpiA}, \Delta \mathrm{gldA}, \mathrm{Apr}^{\mathrm{r}}$

$\Delta$ mgsA, $\Delta$ tpiA, $\Delta$ gldA, $\Delta$ dhaD, Apr ${ }^{r}, \operatorname{Str}^{r}$

$\Delta$ dhaD, $\Delta$ tpiA, $A$ r $^{r}$

$\Delta$ budA, $\Delta$ tpiA, Apr ${ }^{r}, \operatorname{Str}^{r}$

$\Delta$ gapA, Apr ${ }^{r}$

$\Delta \mathrm{gapC}, \mathrm{Apr}^{\mathrm{r}}$

$\Delta$ gapA, $\Delta$ gapC, Apr ${ }^{r}, \operatorname{Str}^{r}$

$\Delta$ dhak, $\Delta$ tpiA, Apr ${ }^{r}$

$\Delta$ dhak1, $\Delta$ tpiA, Apr ${ }^{r}$

$\Delta$ dhak2, $\Delta$ tpiA, Apr ${ }^{r}$

$\Delta$ dhak3, $\Delta$ tpiA, Apr ${ }^{r}$

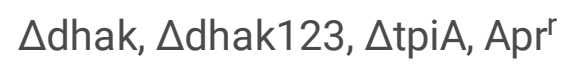

Apr', aac(3)IV with FRT sites, 4334 bp

Str', aadA with FRT sites, 4337 bp

$\mathrm{Kan}^{\mathrm{r}}$, lacl $^{\mathrm{Q}}$, tac, $5118 \mathrm{bp}$

$\mathrm{Kan}^{\mathrm{r}}$, carries $h d p A, 5.9 \mathrm{kp}$

$\mathrm{Kan}^{r}$, carries $\lambda$-Red genes (gam, bet, exo)

$7.1 \mathrm{kp}$
Reference or source

Lab stock

Lab stock

[7]

This work

This work

This work

This work

This work

This work

This work

This work

This work

This work

This work

This work

This work

This work

[26]

[26]

[27]

This work

[28]

[28] 


\begin{tabular}{|c|c|c|}
\hline & $\begin{array}{l}\text { Kan }^{r} \text {, carries the yeast FLP recom } \\
\text { gene } 6.3 \mathrm{kp}\end{array}$ & \\
\hline pMD18-T-simple & $\mathrm{Amp}^{r}$, TA cloning vector, $2692 \mathrm{bp}$ & Takara \\
\hline pMD18-T- $\Delta$ tpiA & $A m p^{r}, A p r^{r}$, carries part of $t p i A$ & This work \\
\hline pMD18-T- $\Delta$ mgsA & $\mathrm{Amp}^{r}, \mathrm{Str}^{\mathrm{r}}$, carries part of $m g s A$ & This work \\
\hline pMD18-T- $\Delta$ gldA & $A m p^{r}, A p r^{r}$, carries part of $g / d A$ & This work \\
\hline pMD18-T- $\Delta$ dhaD & $A m p^{r}, \operatorname{Str}^{r}$, carries part of $d h a D$ & This work \\
\hline pMD18-T- $\Delta$ gapA & $A m p^{r}, A p r^{r}$, carries part of gapA & This work \\
\hline pMD18-T- $\Delta$ gapC & $A m p^{r}, A p r^{r}$, carries part of gapC & This work \\
\hline
\end{tabular}

\section{Figures}


Figure 1

Glycerol catabolic pathway, reverse pathway, and related pathways of K. pneumoniae 

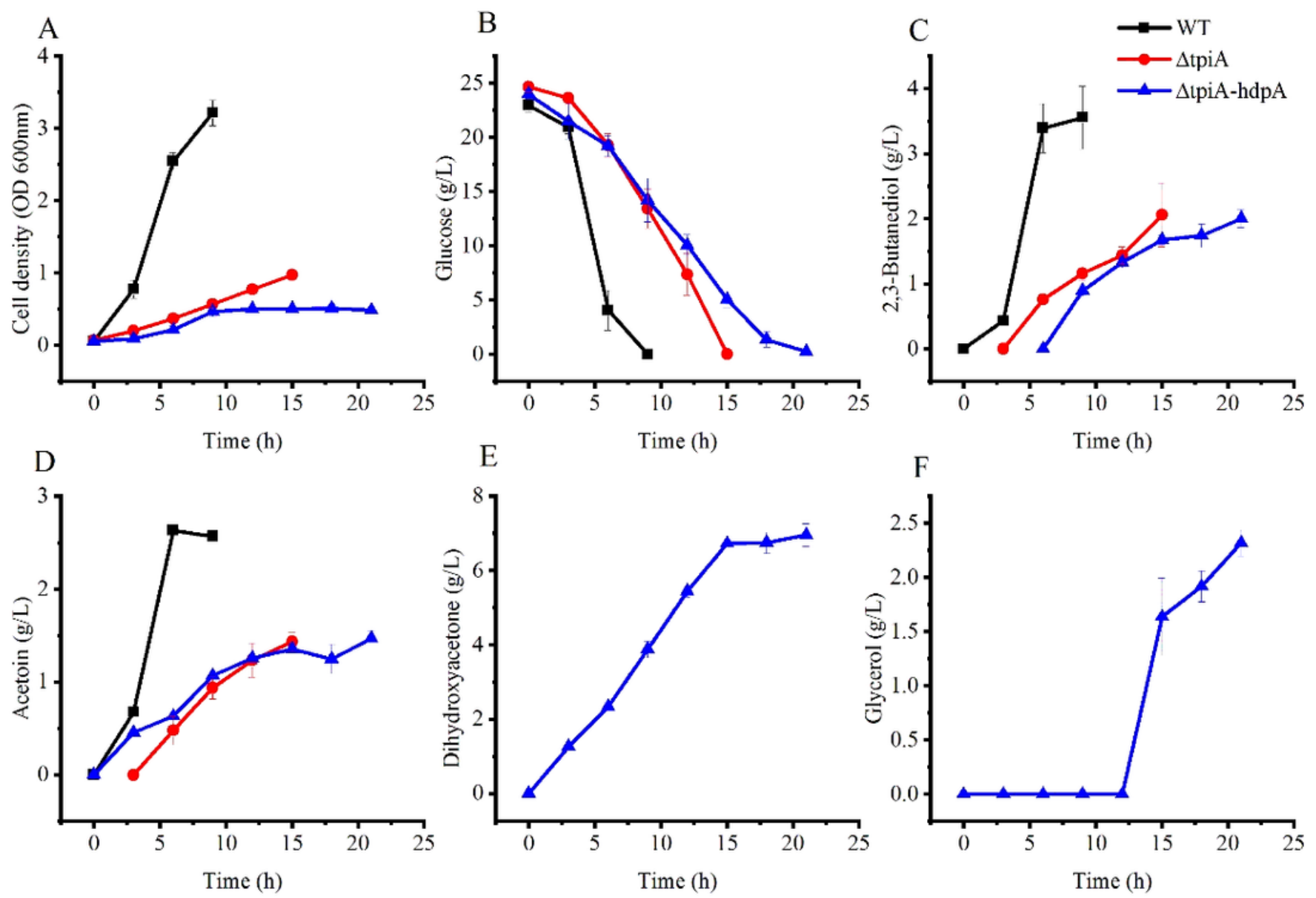

Figure 2

Growth and product formation of wild type K. pneumoniae, Kp $\Delta$ tpiA and $\mathrm{Kp} \Delta \mathrm{tpiA-hdpA}$ grown in shake flasks. WT: Wild-type; $\Delta$ tpiA: Kp $\Delta$ tpiA; $\Delta$ tpiA-hdpA: Kp $\Delta$ tpiA-hdpA. Data points are the average of $\mathrm{n}=3$; error bars represent standard error about the mean. 

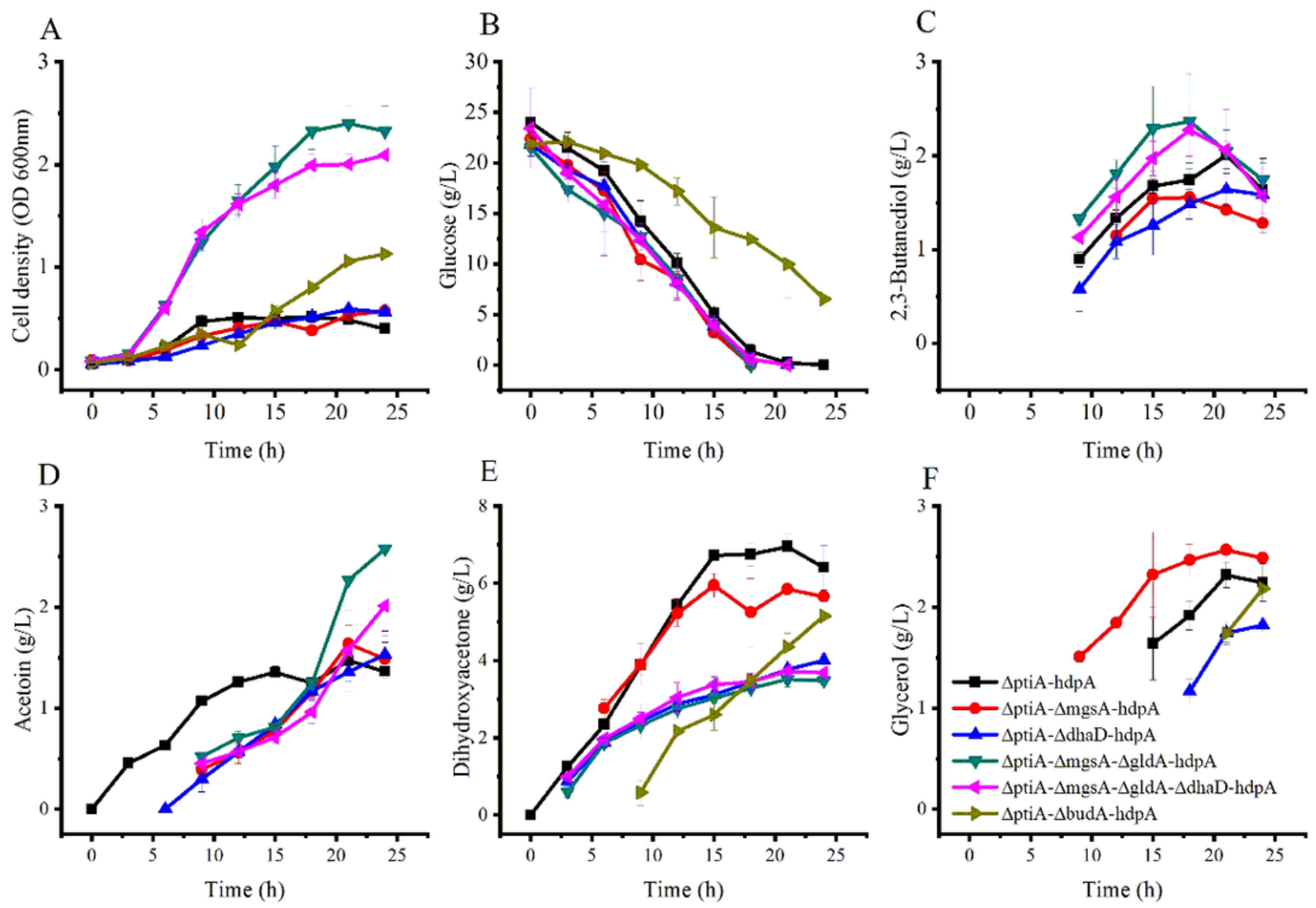

Figure 3

Growth and product formation of K. pneumoniae strains grown in shake flasks for reduce by-products of DHA production. $\Delta$ tpiA-hdpA: Kp $\Delta$ tpiA-hdpA; $\Delta$ tpiA- $\Delta$ mgsA-hdpA: Kp $\Delta$ tpiA- $\Delta$ mgsA-hdpA; $\Delta$ tpiA- $\Delta$ dhaDhdpA: Kp $\Delta$ tpiA- $\Delta$ dhaD-hdpA; $\Delta$ tpiA- $\Delta$ mgsA- $\Delta$ gldA-hdpA: Kp $\Delta$ tpiA- $\Delta$ mgsA- $\Delta$ gldA-hdpA; $\Delta$ tpiA- $\Delta$ mgsA$\Delta$ gldA- $\Delta$ dhaD-hdpA: Kp $\Delta$ tpiA- $\Delta$ mgsA- $\Delta$ gldA- $\Delta$ dhaD-hdpA; $\Delta$ tpiA- $\Delta$ budA-hdpA: Kp $\Delta$ tpiA- $\Delta$ budA-hdpA; Data points are the average of $n=3$; error bars represent standard error about the mean. 

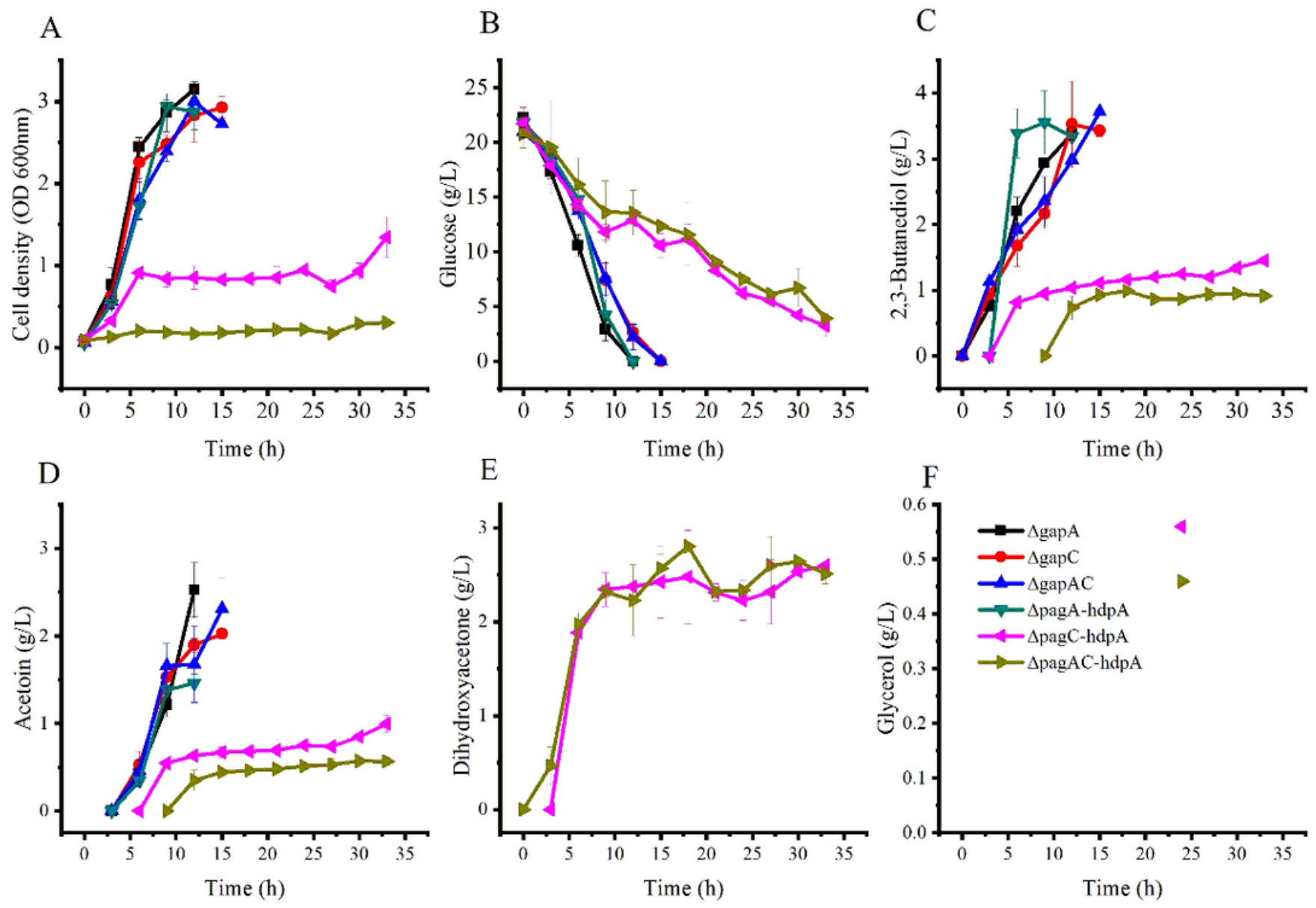

\section{Figure 4}

DHA production by glyceraldehyde-3-phosphate dehydrogenase disruption strains of K. pneumoniae grown in shake flasks. $\Delta$ gapA: Kp $\Delta$ gapA; $\Delta$ gapC: Kp $\Delta$ gapC; $\Delta$ gapAC: Kp $\Delta$ gapC- $\Delta$ gapA; $\Delta$ gapA -hdpA: Kp $\Delta$ gapA-hdpA; $\Delta$ gapC-hdpA: Kp $\Delta$ gapC-hdpA; $\Delta$ gapAC-hdpA :Kp $\Delta$ gapC- $\Delta$ gapA-hdpA. Data points are the average of $n=3$; error bars represent standard error about the mean. 

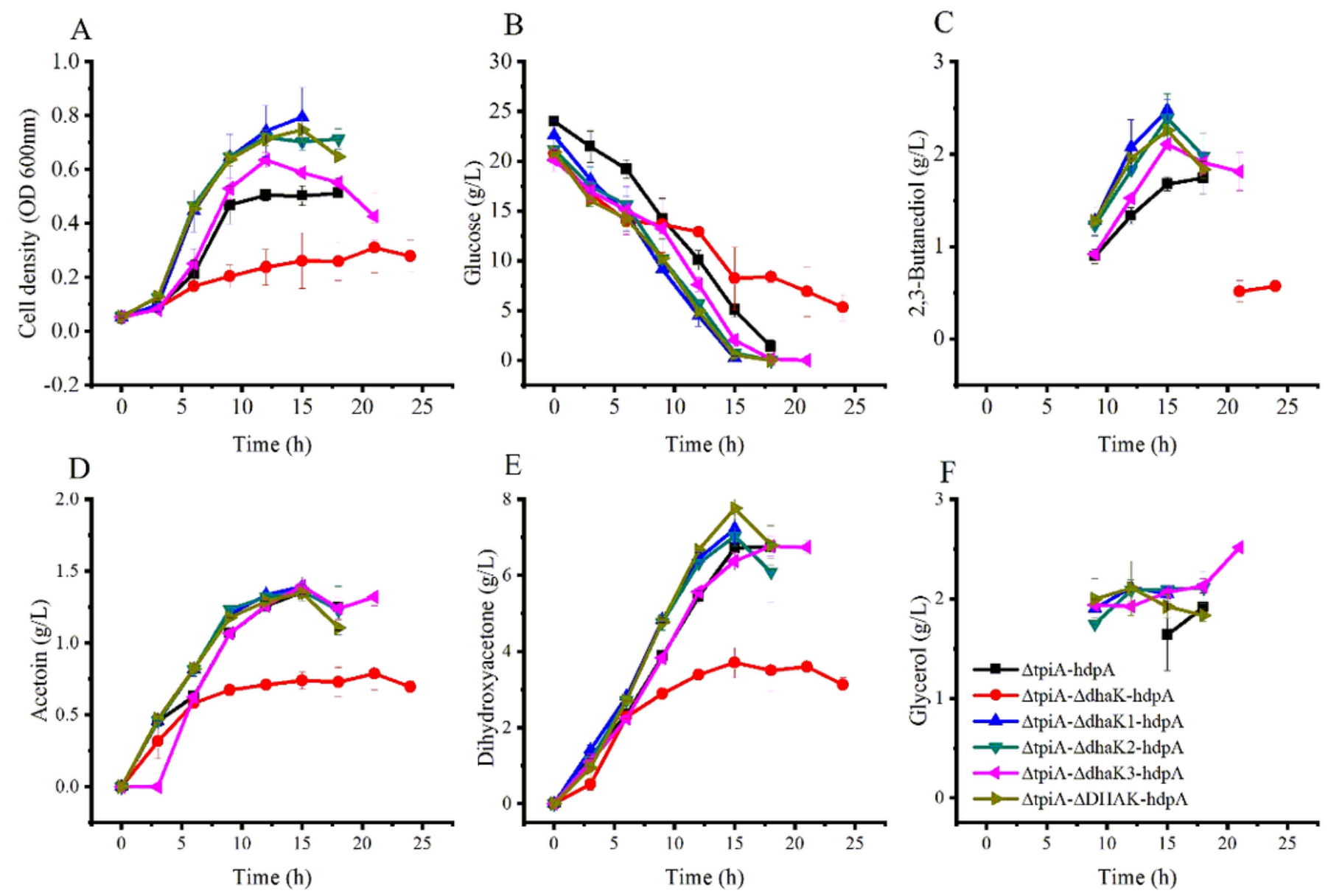

\section{Figure 5}

Growth and product formation of K. pneumoniae strains that knocked out genes that encoding isoenzymes of DHA kinases in shake flasks. $\triangle$ tpiA-hdpA: Kp $\triangle$ ptiA-hdpA; $\triangle$ tpiA- $\Delta$ dhaK-hdpA: Kp $\Delta$ ptiA$\Delta$ dhaK-hdpA; $\Delta$ tpiA- $\Delta$ dhaK1-hdpA: Kp $\Delta$ ptiA- $\Delta$ dhaK1-hdpA; $\Delta$ tpiA- $\Delta$ dhaK2-hdpA: Kp $\Delta$ ptiA- $\Delta$ dhaK2-hdpA; $\Delta$ tpiA- $\Delta$ dhaK3-hdpA: Kp $\Delta$ ptiA- $\Delta$ dhaK3-hdpA; $\Delta$ tpiA- $\Delta$ DHAK-hdpA: Kp $\Delta$ ptiA- $\Delta$ DHAK-hdpA. Data points are the average of $n=3$; error bars represent standard error about the mean. 

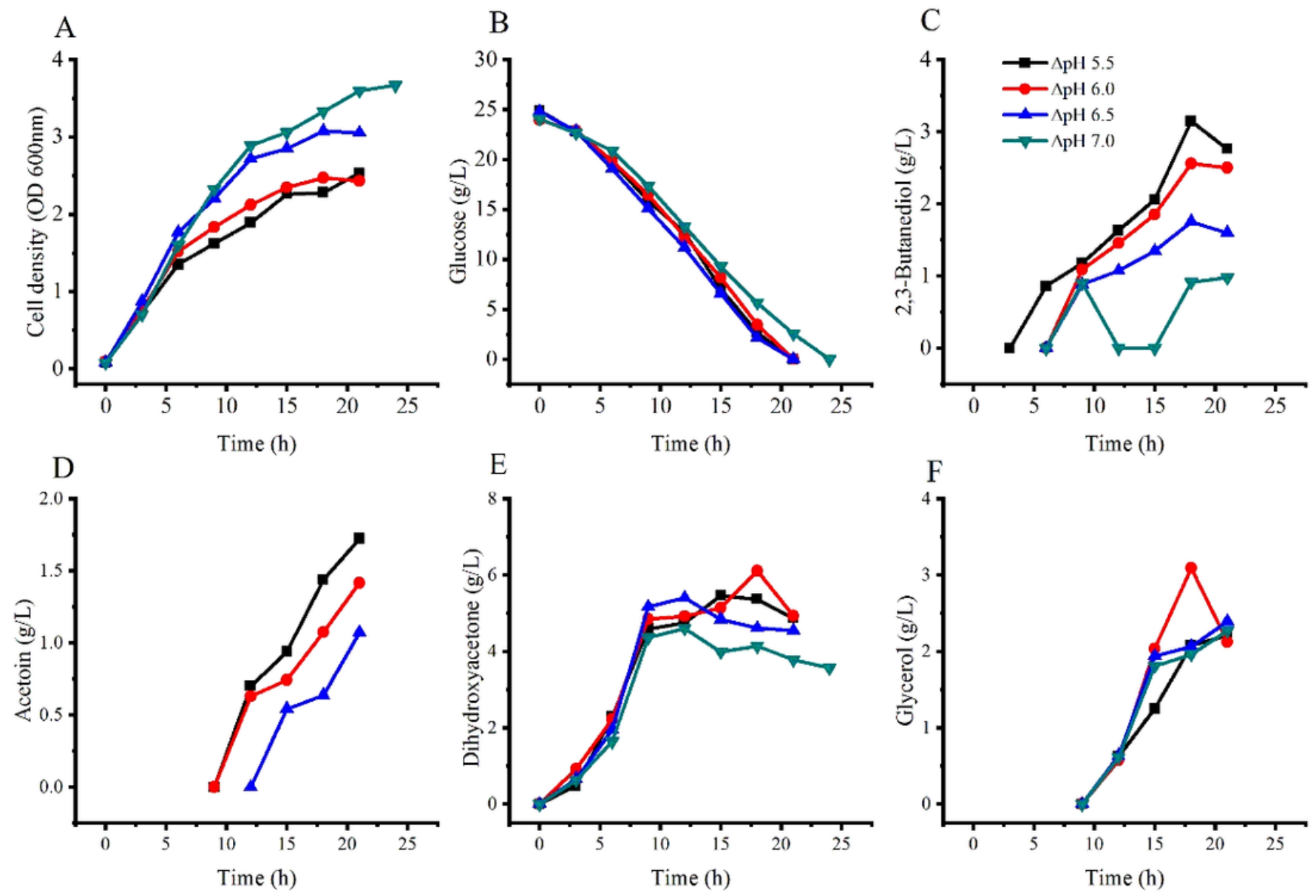

Figure 6

Growth and product formation of $\mathrm{Kp} \Delta \mathrm{tpiA}-\triangle \mathrm{DHAK}$-hdpA in bioreactors at different culture $\mathrm{pH}$. The strain was grown in stirred tank bioreactors operated at an agitation rate of $250 \mathrm{rpm}$ and an aeration rate of 2 L/min. 

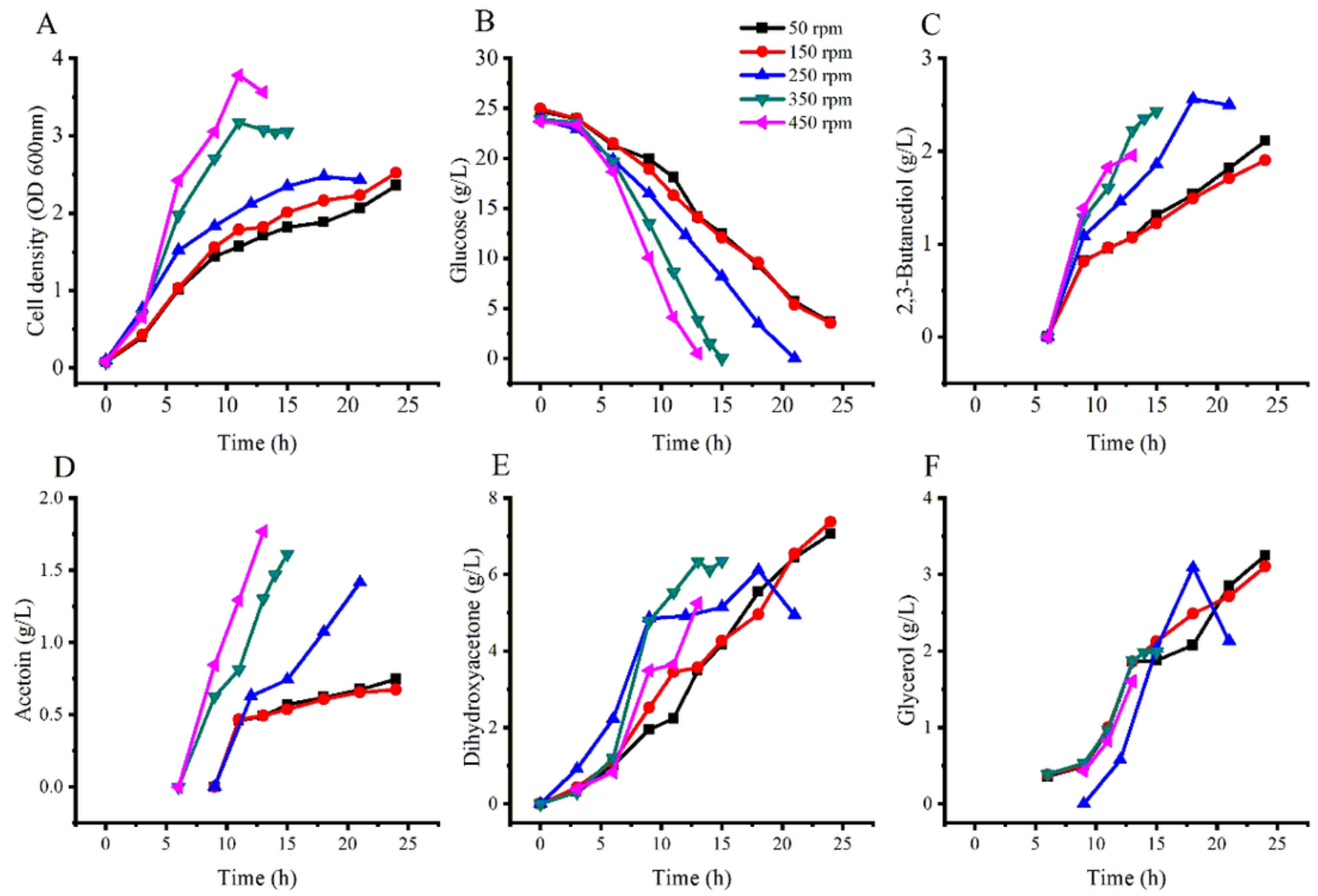

Figure 7

Growth and product formation of Kp $\Delta$ tpiA- $\triangle D H A K$-hdpA in bioreactors with different stirring rates. The strain was grown in stirred tank bioreactors and the aeration rate set at $2 \mathrm{~L} / \mathrm{min}$, culture pH 6.0. $(250 \mathrm{rpm}$ data were the same shown in Fig. 6) 

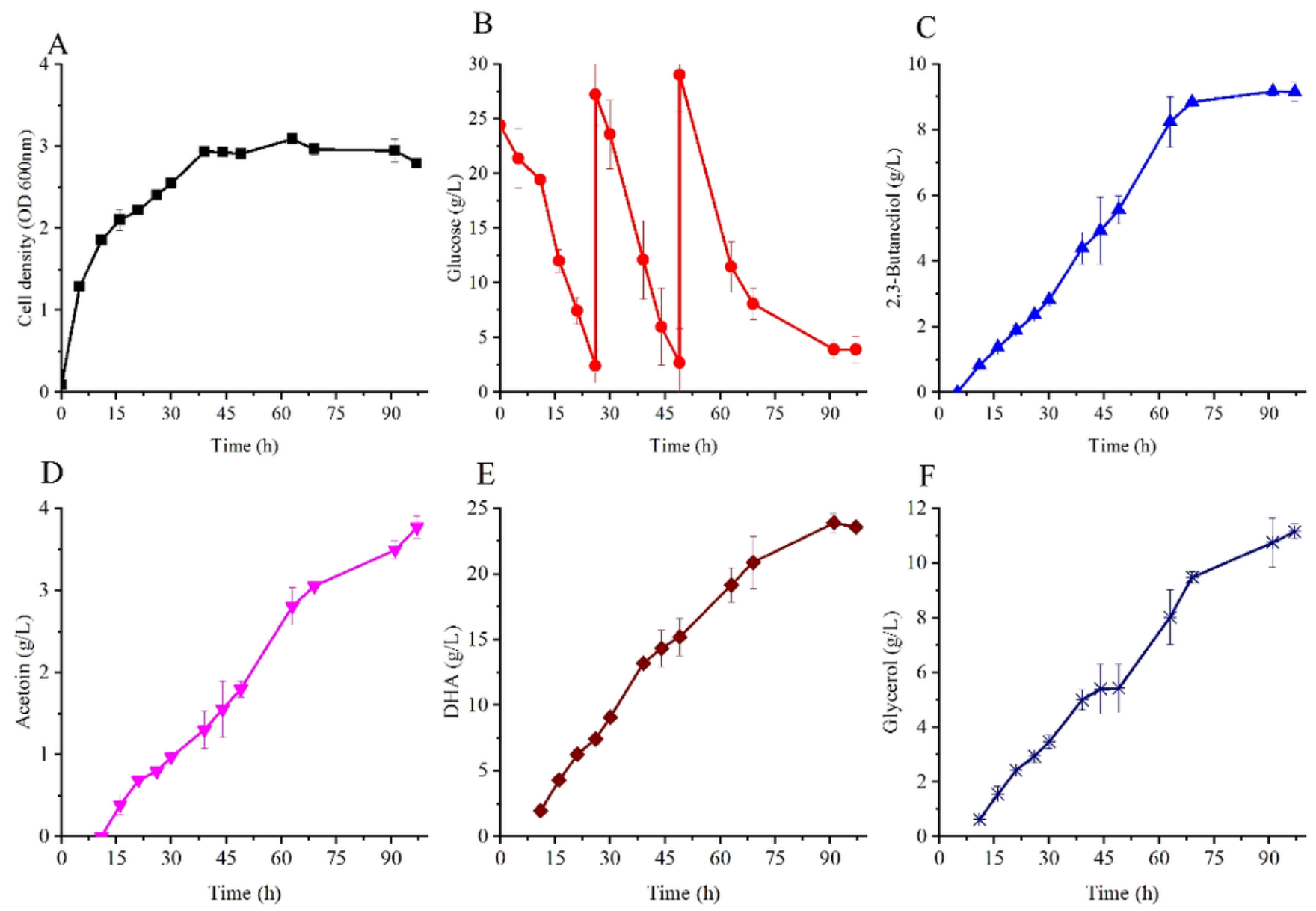

Figure 8

Growth and product formation of $\mathrm{Kp} \Delta \mathrm{tpiA}-\triangle \mathrm{DHAK}$-hdpA in fed batch fermentation. Cells were grown in a stirred tank bioreactor operated at aeration rate of $2 \mathrm{~L} / \mathrm{min}$. In the first 6 hours the culture $\mathrm{pH}$ and stirring rate were set at 7.0 and $400 \mathrm{rpm}$. After 6 hours the culture $\mathrm{pH}$ and stirring rate were set at 6.0 and 150 rpm. Glucose was fed at 26 and 49 hours of cultivation. Data points are the average of $n=3$; error bars represent standard error about the mean.

\section{Supplementary Files}

This is a list of supplementary files associated with this preprint. Click to download.

- Supplementary.docx 